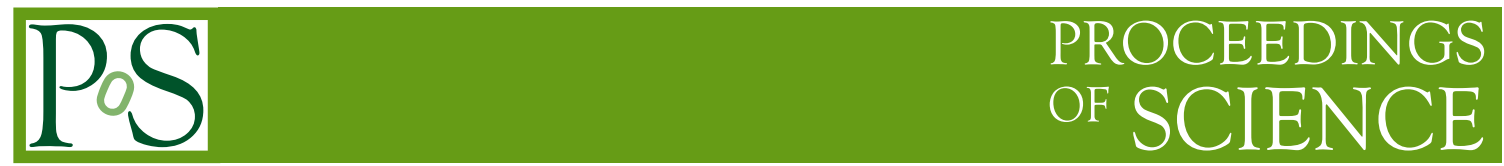

\title{
Searches for heavy resonances decaying to Higgs bosons at ATLAS \& CMS
}

\author{
$\mathbf{K e} \mathbf{L i}^{1, *}$ \\ Department of Physics, University of Washington, \\ Seattle, WA, United States of America \\ E-mail: ke.1i@cern.ch
}

This document highlights a selection of recent searches for new heavy resonances using the decay to Higgs bosons from ATLAS and CMS Collaborations. The results are obtained based on the proton-proton collision data collected during Run 2 of the LHC at a center-of-mass energy of 13 TeV. No significant deviations from the Standard Model expectations are observed.

The Eighth Annual Conference on Large Hadron Collider Physics-LHCP2020

25-30 May, 2020

online

${ }^{1}$ For the ATLAS and CMS Collaborations

* Speaker 


\section{Introduction}

After the Higgs $(\mathrm{H})$ boson was discovered by the ATLAS [1] and CMS [2] Collaborations, one of the major focuses is the search for new physics beyond the Standard Model (SM). In many theoretical models, new resonances are predicted and could couple to Higgs bosons.

This proceeding presents five recent searches for new heavy resonances decaying to Higgs bosons. The di-Higgs $(\mathrm{HH})$ and vector-boson association $(\mathrm{VH})$ processes have been used to investigate different benchmark scenarios, including the spin-0 scalar in the two-higgs-doublet (2HDM) model [3], spin-1 $W^{\prime} / Z^{\prime}$ in the heavy vector triplet (HVT) model [4], and spin-2 graviton in the bulk Randall-Sundrum (RSG) model [5]. The data samples used in the searches were collected with the ATLAS and CMS detectors during 2015 to $2018 p p$ collision runs at $\sqrt{s}=13 \mathrm{TeV}$ and correspond to total integrated luminosity up to $139 \mathrm{fb}^{-1}$.

\section{General search strategy}

For $p p$ collisions, the new heavy resonance can be produced via gluon-gluon fusion ( $\mathrm{ggF})$, vector-boson fusion (VBF) and vector-boson association processes. Thus a series of searches is conducted to cover different signal signatures. The ggF and VBF processes are used to search for spin- 0 and spin- 2 resonances, while $\mathrm{VH}$ is used to search for spin-1 resonances.

Since all the studies are performed with at least one Higgs boson, the ideal decay channel for this search is $H \rightarrow b b$, which has the largest branch fraction. As for the backgrounds for all of these searches, the MC simulation and data-driven methods are used to estimate the contributions.

To search for resonances with extremely high mass, the Higgs or vector bosons could be highly boosted. Therefore the main experimental challenge is the reconstruction and identification of the boosted object. There are many advanced techniques that have been used to study the boosted object, more details are described in Ref. [6].

\section{Boosted $\mathrm{HH}$ results}

The recent result from CMS for HH resonant search is conducted with $35.9 \mathrm{fb}^{-1} p p$ collision data. This search is designed for the resonance with mass greater than $0.8 \mathrm{TeV}$, therefore the Higgs bosons tends to have high transverse momentum and should be reconstructed from boosted objects in detector. The $H H \rightarrow b b W W^{*}, W W^{*} \rightarrow q q l$ channel is used and boosted $W W^{*}$ candidate is reconstructed by a large-radius jet and a nearby lepton. The boosted $H \rightarrow b b$ is reconstructed by another large radius jet and both the subjets are tagged as $b$-jet. No derivation from SM prediction is observed, and the upperlimits at $95 \%$ confidence level (CL) on the product of cross section and branching fraction range from $123 \mathrm{fb}$ at $0.8 \mathrm{TeV}$ to $8.3 \mathrm{fb}$ at $3.5 \mathrm{TeV}$ for spin- 0 resonance, and from $103 \mathrm{fb}$ at $0.8 \mathrm{TeV}$ to $7.8 \mathrm{fb}$ at $3.5 \mathrm{TeV}$ for spin-2 resonance, as shown in Figure 1.

At ATLAS, the $p p$ collison data at $\sqrt{s}=13 \mathrm{TeV}$ with a corresponding luminosity of $139 \mathrm{fb}^{-1}$ has been first fully investigated for $H H$ resonant search. The search is performed with $b b \tau \tau$ channel and designed for the resonance with mass greater than $1 \mathrm{TeV}$. A Boosted-Decision-Tree (BDT) with tracking, vertexing and calorimeter information is used to identify the boosted hadronic $\tau \tau$ [7], and the performance improvement compared to the previous approach is shown in Figure 2 (left). The 

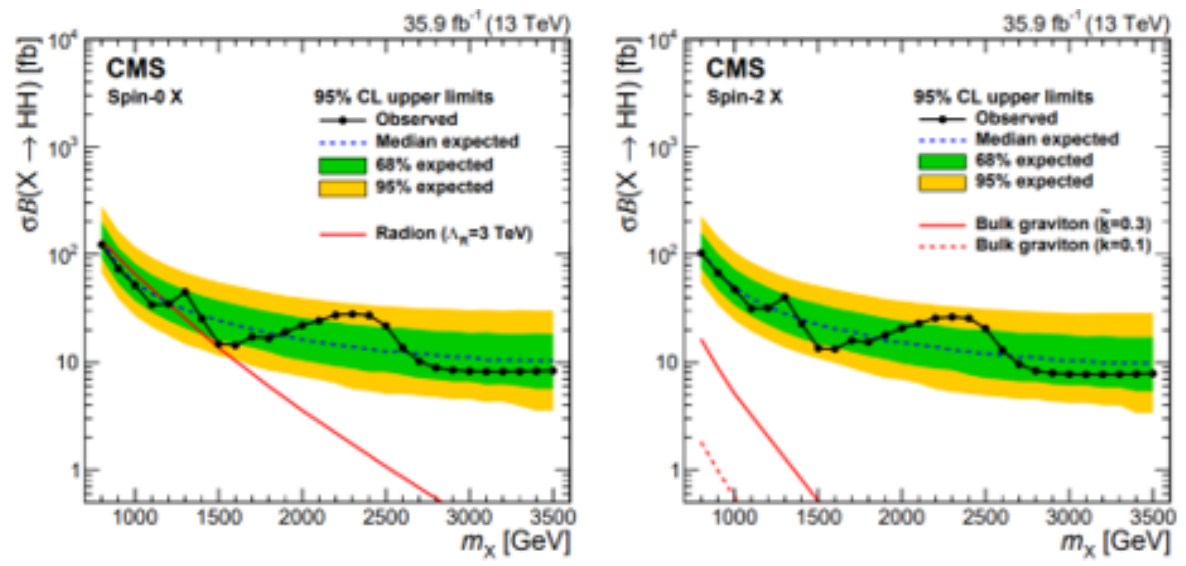

Figure 1: Upperlimit on the product of cross section times branch fraction for spin-0 (left) and spin-2 (right) resonance.

boosted $H \rightarrow b b$ is reconstructed with a large-radius jet and the two variable-radius (VR) subjets are tagged as $b$ jets No significant deviations from the SM predictions are observed, and upperlimits are set on the cross section of a heavy, narrow, spin- 0 resonance as shown in Figure 2 (right). The observed upperlimits range from 88 to $46 \mathrm{fb}$ for resonant mass at 1.2 and $3.0 \mathrm{TeV}$.


Figure 2: Right: Reconstruction efficiency for hadronic $\tau \tau$ from the BDT tagger. Left: Expected and observed $95 \%$ CL upper limits on the production cross-section of a spin-0 resonance decaying into a pair of Higgs bosons. The discontinuities in the limits around $2.5 \mathrm{TeV}$ caused by the different requirements applied on visible mass.

\section{Resolved HH results}

Besides the resonant search with boosted objects, ATLAS and CMS have conducted the searchs for resonance below $1 \mathrm{TeV}$ with resolved objects. The ggF $H H \rightarrow b b Z Z^{*}$ and VBF $H H \rightarrow b b b b$ channels are investigated with the data from CMS and ATLAS, respectively. The $Z Z^{*} \rightarrow j j l l$ or $l l v v(l=e, \mu)$ channel is used and the upperlimits on the product of cross sections of spin- 0 resonance [8] is shown in Figure 3 (left). The VBF process has unique sensitivity to di-vectorboson-di-Higgs-boson quartic coupling compared with ggF and also can be used to search for $\mathrm{HH}$ resonant. At ATLAS, due to the inefficiency b-jet trigger during 2016 data taking, this channel is 

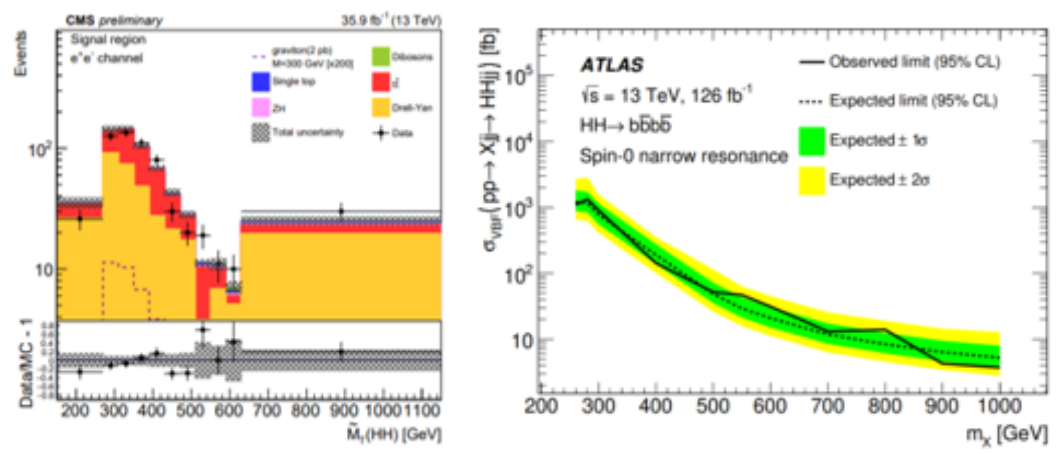

Figure 3: Expected (black dashed line) and observed (black solid line) limits on the cross section of resonant $\mathrm{HH}$ production as a function of the mass of the resonance from the ggF $b b Z Z^{*}$ (left) and $\mathrm{VBF} b b b b$ channel (right).

analyzed with a $126 \mathrm{fb}^{-} 1$ dataset and the upperlimits on the product cross sections (Figure 3 (right)) for resonance below $1 \mathrm{TeV}$ in VBF process is obtained for the first time [9].

\section{Boosted VH results}

In the context of HVT model, the spin- $1 W^{\prime} Z^{\prime}$ interacts with quarks and the Higgs field with coupling strength of $g_{q}$ and $g_{H}$, respectively. Therefore it could be searched with WH and ZH channels. ATLAS has performed a corresponding search with the $139 \mathrm{fb}^{-1} p p$ collision data. The search is designed for resonance with a narrow width and a mass above $1.5 \mathrm{TeV}$, thus both $\mathrm{V}$ and $\mathrm{H}$ bosons tend to be boosted objects in detector. The dominant decay channel, $V H \rightarrow q q b b$, is utilized with two large-radius jets. The jet substructure techniques and VR subjet-based $b$ tagging are used to distinguish $\mathrm{V}$ and $\mathrm{H}$ jets from backgrounds. No significant derivation from SM prediction is observed and the upperlimits on cross sections for $p p \rightarrow W^{\prime} \rightarrow W H$ and $p p \rightarrow Z^{\prime} \rightarrow Z H$ range from $6.8 \mathrm{fb}$ to $0.53 \mathrm{fb}$ and from $8.7 \mathrm{fb}$ to $0.53 \mathrm{fb}$, respectively [10]. The lower limit on the mass of $W^{\prime}\left(Z^{\prime}\right)$ is calculated to be $2.90(2.20) \mathrm{TeV}$ and $3.20(2.65) \mathrm{TeV}$ in the scenario of weekly coupled HVT model A $\left(g_{q}=-0.55\right.$ and $\left.g_{H}=-0.56\right)$ and strongly coupled HVT model B $\left(g_{q}=0.14\right.$ and $\left.g_{H}=-2.9\right)$, respectively. The exclusions of the couplings can be derived from the above results and shown in Figure 4.

\section{Summary}

ATLAS and CMS has a suite of $\mathrm{HH}$ and $\mathrm{VH}$ resonant searches across a large mass range in $b b W W^{*}, b b \tau \tau, b b Z Z^{*}, b b b b$ and $q q b b$ channels. In particular, the boosted $H H \rightarrow b b \tau \tau$ and $V H \rightarrow q q b b$ channels are investigated with the $p p$ collision data at $\sqrt{s}=13 \mathrm{TeV}$ during the LHC full Run2 period for the first time. No significant sign of heavy resonant has been observed so far, and the corresponding upperlimits on product cross sections are reported. The sensitivity is improved remarkably benefited from the increased integrated luminosity and better-performing techniques. Discovery potential for new resonance will be further enhanced with more data and new techniques at HL-LHC. 



Figure 4: Upperlimits on the couplings for the WH (left) and ZH (right) channels with resonant masses of 2,3 and $4 \mathrm{TeV}$.

\section{References}

[1] ATLAS Collaboration, Observation of a new particle in the search for the Standard Model Higgs boson with the ATLAS detector at the LHC, Phys. Lett. B 716 (2012) 1, arXiv:1207.7214[hep-ex]

[2] CMS Collaboration, Observation of a new boson at a mass of $125 \mathrm{GeV}$ with the CMS experiment at the LHC, Phys. Lett. B 716, (2012) 30, arXiv:1207.7235[hep-ex]

[3] G. C. Branco et al., Theory and phenomenology of two-Higgs-doublet models, Phys. Rept. 516, (2012) 1, arXiv: 1106.0034[hep-ph].

[4] D. Pappadopulo, A. Thamm, R. Torre, A. Wulzer, Heavy vector triplets: bridging theory and data, JHEP 9 (2014) 60, arXiv:1402.4431 [hep-ph]

[5] K. Agashe, H. Davoudiasl, G. Perez, A. Soni, Warped gravitons at the CERN LHC and beyond, Phys. Rev. D 76 (2007) 036006, arXiv:hep-ph/0701186.

[6] A. Abdesselam et al. Boosted Objects: A Probe of Beyond the Standard Model Physics, Eur. Phys. J. C 71 (2011) 1661, arXiv:1012.5412 [hep-ph]

[7] ATLAS Collaboration, Reconstruction and identification of boosted di- $\tau$ systems in a search for Higgs boson pairs using $13 \mathrm{TeV}$ proton-proton collision data in ATLAS, CERN-EP-2020118, arXiv:2007.14811 [hep-ex]

[8] CMS Collaboration, Search for the resonant production of a pair of Higgs bosons decaying to the bbZZ final state, Phys. Rev. D 102 (2020) 032003, arXiv:2006.06391 [hep-ex]

[9] ATLAS Collaboration, Search for the $H H \rightarrow b \bar{b} b \bar{b}$ process via vector-boson fusion production using proton-proton collisions at $\sqrt{s}=13 \mathrm{TeV}$ with the ATLAS detector, JHEP 07 (2020) 108, arXiv:2001.05178 [hep-ex] 
[10] ATLAS Collaboration, Search for resonances decaying into a weak vector boson and a Higgs boson in the fully hadronic final state produced in proton-proton collisions at $\sqrt{s}=13 \mathrm{TeV}$ with the ATLAS detector, CERN-EP-2020-073 , arXiv:2007.05293 [hep-ex] 